\title{
Effect of simultaneous surgical treatment in scoliosis associated with intraspinal abnormalities: A retrospective study
}

\author{
KAI WANG ${ }^{*}$, FENG SHANG ${ }^{*}$, FENG-ZENG JIAN and HAO WU \\ Department of Neurosurgery, Xuanwu Hospital of Capital Medical University, Beijing 100053, P.R. China
}

Received March 26, 2019; Accepted December 20, 2020

DOI: $10.3892 /$ etm.2020.9236

\begin{abstract}
To evaluate the outcomes of single-stage surgical treatment for spinal deformity and coexisting intraspinal pathologies, 12 patients who underwent single-stage surgical treatment for spinal deformity and co-existing intraspinal abnormalities between October 2016 and January 2017 were enrolled in the present study. Treatment for intraspinal abnormalities, posterior correction, osteotomy and internal fixation were performed simultaneously. The clinical and radiological outcomes, surgical details, complications and postoperative outcomes were evaluated. The mean fusion length was 11.0 \pm 2.8 . Both scoliosis Cobb angle (pre-surgery $65.9 \pm 13.4$ vs. post-surgery $21.7 \pm 9.4$ ) and kyphosis (pre-surgery $71.1 \pm 19.5$ vs. post-surgery $31.4 \pm 10.4$ ) were significantly improved post-surgery. Tethered cords were released and epidermoid cysts, gangliogliomas, meningiomas and lipomas were resected. Muscle strength in all patients was improved. The muscular tone of 8 patients was improved. No severe complications occurred postoperatively. None of the patients experienced deterioration in their neurological status nor loss of correction during the 12-24 months' follow-up. The simultaneous surgical treatment for spinal deformity and intraspinal pathology seems to be a safe and effective approach. Neurological deficits were improved postoperatively. Osteotomy produces satisfactory correction results.
\end{abstract}

\section{Introduction}

A previous study has reported that $20-58 \%$ of patients with congenital scoliosis may suffer from intraspinal abnormalities at the same time $(1,2)$. Intraspinal abnormalities may contribute to many problems, such as neurological dysfunction and

Correspondence to: Dr Hao Wu, Department of Neurosurgery, Xuanwu Hospital of Capital Medical University, 45 Changchun Street, Beijing 100053, P.R. China

E-mail: 13901397527@139.com

*Contributed equally

Key words: intraspinal pathology, scoliosis, simultaneous surgical treatment, osteotomy maldistribution of trunk muscle strength, which may further induce neuromuscular scoliosis (3). Neurological dysfunction resulting from intraspinal abnormalities can lead to continuous development of scoliosis, which eventually aggravates neurological dysfunction. Treatment of intraspinal abnormalities, as well as correction of scoliosis, is required for these patients. To improve nerve function and reduce surgery-related complications, a two-stage surgical strategy is considered typical treatment for this disease. First, intraspinal abnormalities are treated by a neurosurgeon. Second, corrective surgery is performed by an orthopedic surgeon 3-6 months after the first-stage surgery. This surgical procedure can not only avoid over-traction of the spinal cord induced by intraspinal abnormalities during corrective surgery, but also allows for examination of nerve function and the identification of causes of complications during the second surgery $(4,5)$.

Nevertheless, there are some limitations to this strategy: i) This procedure contains more than one surgery and exposes the patients to the risks of multiple operations and anesthesia; ii) the primary surgery can destroy the anatomical markers, increasing the difficulty of the second-stage surgery; iii) the adhesions preformed in the primary surgical site can cause difficulties for osteotomy and hemivertebrectomy, and also increases the risk of iatrogenic neural element injury. In addition, scoliosis of more than 40 can still progress following treatment of intraspinal abnormalities, which requires further corrective surgery (6).

With the development of neuroelectrophysiological monitoring technology, it is possible to monitor changes in nerve function (7). To avoid the disadvantages of two-stage surgery, single-stage surgery for intraspinal abnormalities and scoliosis has recently been performed in some institutions $(2,6)$. Thus, the purpose of the present study was to evaluate the safety and efficacy of single-stage surgical treatment for spinal deformity and coexisting intraspinal pathologies.

\section{Materials and methods}

Subjects. A total of 12 consecutive patients with intraspinal abnormalities who underwent single-stage surgery between October 2016 and September 2017 at the Xuanwu Hospital of Capital Medical University (Beijing, China) were included in the current study (7 males and 5 females). Inclusion criteria included: i) Scoliosis with intraspinal lesions; ii) coronal Cobb angle $>40$ or sagittal kyphosis deformity; and iii) single-stage 
surgery for scoliosis/kyphosis deformity and intraspinal lesions. Exclusion criteria included: i) Coronal Cobb angle $<40$ and no kyphosis deformity in the sagittal plane; and ii) staged surgery for intraspinal lesions and scoliosis. The mean age was $29.5 \pm 13.7$ years (range, $11-52$ years). Progressive scoliosis was observed in all the 12 patients, and 5 of the 12 patients were diagnosed with congenital scoliosis, including 1 wedge-shaped vertebra, 1 malsegmentation, 1 wedge-shaped vertebra combined with malsegmentation and 2 hemivertebra with malsegmentation. In addition, 7 patients were diagnosed with neuromuscular scoliosis. Risser sign (8) was level 2 in 1 patient, level 3 in 2 patients and level 5 in 9 patients. Spinal MRI scanning showed that 7 patients had tethered cord secondary to scoliosis and 6 patients had syringomyelia. Epidermoid cyst was observed in 2 patients and ganglioma was found in 1 patient. Intraspinal lipoma was confirmed in 1 patient and meningioma was observed in 1 patient. The mean

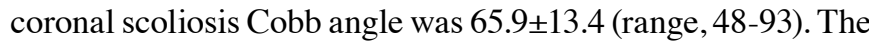
mean sagittal kyphosis angle was $71.1 \pm 19.5$ (range, 44-94). No abnormality was observed in upper extremity myodynamia or muscular tension in the 12 patients. Lower myodynamia of the lower extremity was observed in 10 patients, including level 0 in 4 patients, level 2 in 1 patient, level 3 in 4 patients, level 4 in 1 patient and level 5 in 2 patients. Higher muscular tension of the lower extremity was observed in 8 patients. Preoperative sphincter disturbances were observed in 10 patients. The current study was approved by the ethics committee of the Xuanwu Hospital of Capital Medical University and all the patients or their families gave informed consent and signed a statement to that effect.

Preoperative preparations. The general information including patient name, sex, age and syndromes of nervous system was recorded. Preoperative and postoperative anteroposterior and lateral full spine standing images, CT scanning and 3D reconstruction and MRI scanning of spine were performed to evaluate spine deformity and intraspinal abnormalities. Ultrasound examination of urinary system was performed to assess urinary system deformity. ECG and color Doppler echocardiography were used to evaluate cardiac function. A pulmonary function test (9) was performed to evaluate the progress of ventilatory dysfunction.

Surgical procedure. The fused segments and osteotomy level were decided according to the preoperative radiological results. Following general anesthesia with tracheal intubation, the patient was put in a prone position. The spinous process and vertebral plate surgical levels were carefully explored. Vertebral deformity, fusion and dysplasia were identified attentively and carefully handled to avoid spinal cord injury induced by surgical instruments, especially for patients with spinous process and vertebral plate absence. Following localization of operative segments by intraoperative X-ray, pedicle screws were planted into planned segments. X-ray scanning was performed again to make sure all the screws were in the correct position. As the spinal cord was close to the concave side, pedicle screws on this side were carefully inserted to protect the spinal cord. Following the planting of all the pedicle screws, intraspinal abnormalities were handled under a microscope. Spinal cord untethering was performed for patients with tethered spinal cord. Lesion resection was performed for patients with epidermoid cysts, ganglioma, intraspinal lipoma and meningioma. No special treatment was performed for syringomyelia. After the spinal cord was released, posterior osteotomy and correction were performed under the microscope according to the different spinal deformities. Usually, Schwab grade 2 osteotomy was performed at the apical level of scoliosis or kyphosis, and one segment above and below the apex. For patients with congenital scoliosis or severe kyphosis, Schwab grade 3 or 4 osteotomy was performed according to different deformities or the desired correction. Following osteotomy, a pre-bending rod based on the desired correction was planted. Then compression of the convex side and distraction of the concave side were performed to correct the deformity. The sagittal alignment of the spine should be considered when bending the rod. Finally, mixed autogenous and allograft bone grafting and fusion were performed. During surgery, all the patients were under neuroelectrophysiological monitoring, including somatosensory evoked potential, motor evoked potential and sphincter monitoring. Following surgery, the drainage tube was removed when the daily volume of the drainage was $<20 \mathrm{ml}$, and walking exercise under brace was allowed after the drainage tube was removed. Exercise under brace was required for 3 months postoperatively.

Operation effect evaluation. The operation success rate, operation time, interoperative blood loss, fusion segments, scoliosis and kyphosis Cobb angle, treatment of intraspinal pathology, nervous system function and muscular strength and tension of the upper and lower extremities were recorded. Postoperative complications, including infection, cerebrospinal fluid leakage, spine fixation failure and fracture of pedicle screws and rods, were reviewed. During the follow-up time, complications such as nervous system dysfunction, delayed infection and pseudoarthrosis were recorded.

Statistics analysis. All the data analysis was performed using SPSS 23.0 statistical software (IBM Corp.). A paired sample t-test was performed for the data with a normal distribution. Data with a normal distribution are presented as average value \pm standard deviation Data with a non-normal distribution are presented as the median and interquartile range $[\mathrm{M}(\mathrm{P} 25, \mathrm{P} 75)] . \mathrm{P}<0.05$ was considered to indicate a statistically significant difference.

\section{Results}

Changes of scoliosis and intraspinal abnormalities. The general information of the patients is listed in Table I. Single-stage surgery was performed on all 12 patients with scoliosis and intraspinal abnormalities. The surgical success rate was $100 \%$. The average surgical time was 451 $154 \mathrm{~min}$ (IQR, 241-631 min) and the median value of blood loss was $1,570.8 \pm 770.6 \mathrm{ml}$ (IQR, 400-2,650 ml). The mean length of the fused level was $11.0 \pm 2.8 \mathrm{~cm}$ (IQR, 7-15). The average postoperative coronal Cobb angle was $21.7 \pm 9.4$ (IQR, 12-44) and the sagittal kyphosis was $31.4 \pm 10.4$ (IQR, 10-42), which were significantly different from those before surgery (Fig. 1). The improvements in the coronal and sagittal deformities were calculated to be $67 \pm 11$ and $56 \pm 11 \%$, respectively, according to the data presented 
Table I. General information of the 12 patients.

\begin{tabular}{|c|c|c|c|c|c|c|c|c|}
\hline $\begin{array}{l}\text { Case } \\
\text { no. }\end{array}$ & Sex & Age & Diagnosis & $\begin{array}{l}\text { Intraspinal } \\
\text { pathology }\end{array}$ & $\begin{array}{l}\text { Risser } \\
\text { sign }\end{array}$ & $\begin{array}{c}\text { Blood } \\
\text { loss }(\mathrm{ml})\end{array}$ & $\begin{array}{c}\text { Hospital } \\
\text { stay (days) }\end{array}$ & $\begin{array}{l}\text { Follow-up } \\
\text { (months) }\end{array}$ \\
\hline 01 & Male & 23 & NS & Ganglioma and syringomyelia & 5 & 1,200 & 10 & 24 \\
\hline 02 & Male & 40 & $\mathrm{CS}$ & Epidermoid cyst and syringomyelia & 5 & 700 & 12 & 23 \\
\hline 03 & Female & 24 & $\mathrm{CS}$ & Spinal cord tethering and syringomyelia & 5 & 1,500 & 7 & 23 \\
\hline 04 & Male & 17 & $\mathrm{CS}$ & Spinal cord tethering & 3 & 2,000 & 7 & 22 \\
\hline 05 & Male & 11 & NS & Spinal cord tethering and syringomyelia & 2 & 400 & 6 & 22 \\
\hline 06 & Male & 14 & NS & Spinal cord tethering and intraspinal lipoma & 3 & 2,600 & 7 & 21 \\
\hline 07 & Male & 28 & $\mathrm{CS}$ & Epidermoid cyst & 5 & 1,600 & 5 & 19 \\
\hline 08 & Female & 50 & NS & Spinal cord tethering and syringomyelia & 5 & 700 & 7 & 19 \\
\hline 09 & Female & 52 & NS & Meningioma & 5 & 1,100 & 8 & 18 \\
\hline 10 & Male & 43 & NS & Spinal cord tethering and syringomyelia & 5 & 2,000 & 7 & 17 \\
\hline 11 & Female & 25 & NS & Spinal cord tethering & 5 & 2,650 & 6 & 12 \\
\hline 12 & Female & 27 & $\mathrm{CS}$ & Spinal cord tethering and diastematomyelia & 5 & 2,400 & 5 & 12 \\
\hline
\end{tabular}

NS, neuromuscular scoliosis; CS, congenital scoliosis.

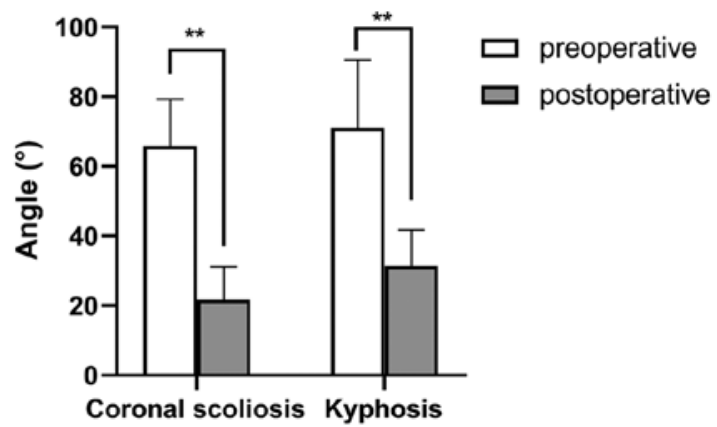

Figure 1. Coronal scoliosis and kyphosis of the patients before and after surgery. ${ }^{* *} \mathrm{P}<0.01 ; \mathrm{n}=9$.

in Table II. For patients with hemivertebra and dyssegmentation, further Schwab grade 3 or 4 osteotomy was performed to release the anterior column and resect the hemivertebra to obtain improved orthopedic effects. In addition, coronal central sacral vertical line decreased from $19.4(+11.3 \mathrm{~mm})$ preoperatively to $8.4(+7.0 \mathrm{~mm})$ postoperatively, and coronal deviation was improved. Sagittal vertical axis was $25.5(+19.3 \mathrm{~mm})$ and $29.7(+9.1 \mathrm{~mm})$ preoperatively and postoperatively, respectively, with no significant sagittal imbalance (data not shown).

Radiographic images. Spinal cord untethering was performed for patients with tethered spinal cord. Lesion resection was performed for patients with epidermoid cyst, ganglioma, intraspinal lipoma and meningioma. No special treatment was performed for syringomyelia. Radiographic photographs of typical case are shown in Figs. 2 and 3. Fig. 2 illustrates the case of a 17-year-old male patient with congenital scoliosis associated with the tethered cord (case no. 4). Thoracic curve and lumbar curve were severe, with trunk shift compensated, shoulder and pelvic obliquity, leg length discrepancy and failure of segmentation of T5-T7 and L1-L2 could be found preoperatively. In the postoperative image without shoe inserts, it can be identified that scoliosis was corrected and the shoulder obliquity was improved, but the pelvic obliquity (the angle between the pelvic coronal reference line and the horizontal reference line) remained and the trunk shift (increased distance between the C7 plumb line and the center sacral vertical line) was decompensated due to the leg length discrepancy (the difference between the higher and lower horizontal reference line of femoral head). In the postoperative image with shoe inserts, it can be identified that that the leg length discrepancy and pelvic obliquity (the angle between the pelvic coronal reference line and the horizontal reference line decreased) were corrected and trunk shift (decreased distance between the $\mathrm{C} 7$ plumb line and the center sacral vertical line) compensated. Fig. 3 illustrates the case of a 27-year-old female diagnosed with congenital scoliosis and tethered spinal cord (case no. 12). Coronal thoracic bending and lumbar bending were observed 3 years before operation, the trunk was basically balanced by compensation, T11 hemivertebra and T11-12 vertebral body were segmented badly (Fig. 3A and C). Sagittal T2 WI MR before surgery indicated spinal cord tethered (Fig. 3E). Preoperative three-dimensional reconstruction CT showed that the T11-12 vertebral body was segmented badly, and the T11 hemivertebra was visible (Fig. 3F). Thick terminal filaments were observed during the operation. After electrophysiological monitoring confirmed the absence of nerves (Fig. 4A), the terminal filaments were cut off (Fig. 4B). The patient was treated with spinal cord tether release, T11 hemivertebra excision and lateral bending correction, and satisfactory results were achieved (Fig. 3B and D).

Complications and functional recovery following surgery. No deterioration of neurological dysfunction was observed following surgery. The postoperative muscle strength of the lower extremities was grade 3 in 2 patients, grade 4 in 5 patients and grade 5 in 5 patients, which were markedly improved compared to preoperative muscle strength. Following surgery, lower muscle tension was observed in the 8 patients with preoperative high muscle tension. The mean stay in hospital 


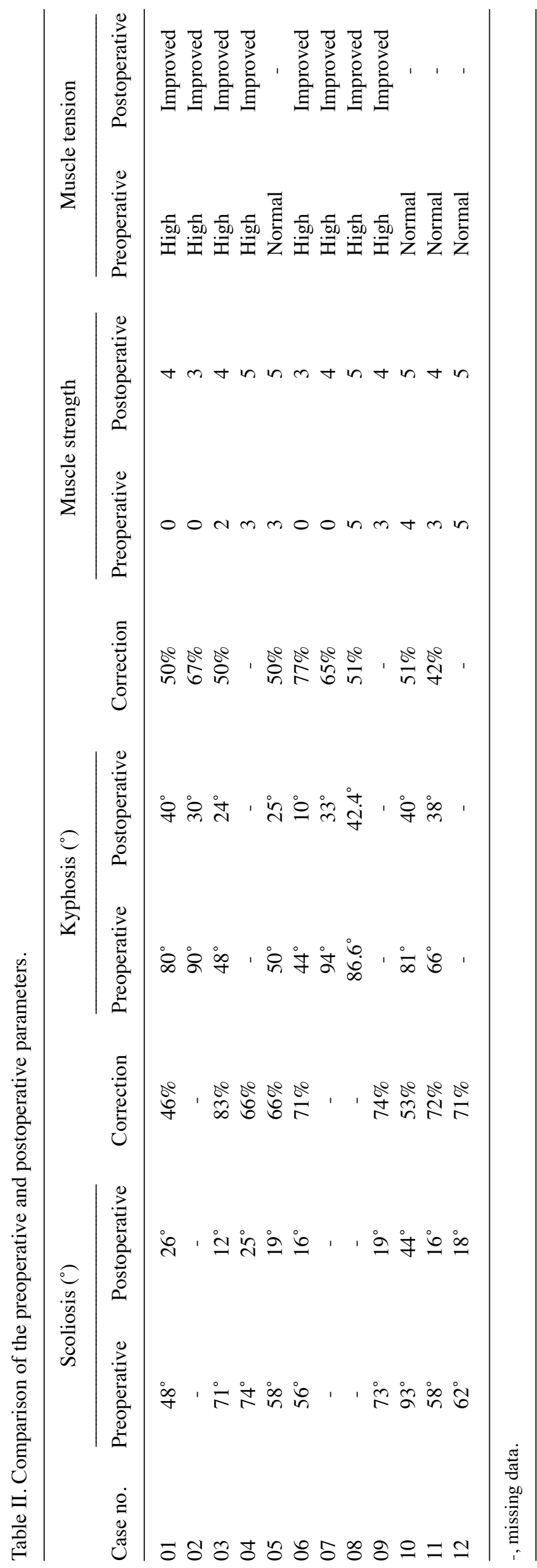

was $7.3 \pm 2.0$ days (range, 5-12 days). No complications, such as infection, cerebrospinal fluid leakage, fixation failure and fracture of rods and screws, was found postoperatively. The mean follow-up time was $19.3 \pm 4.1$ months (range, 12-24 months). No mortality, deterioration of neurological dysfunction, tardive dyskinesia, pseudarthrosis and correction loss was observed during the follow-up time.

\section{Discussion}

Coexisting intraspinal abnormalities can be found in both congenital and neuromuscular scoliosis. Previous studies have reported that intraspinal abnormalities can be observed on preoperative MRI images in $20-58 \%$ patients with congenital scoliosis, including the tethered spinal cord, syringomyelia, intraspinal lipoma and teratoma $(4,10)$. Neuromuscular scoliosis induced by intraspinal abnormalities is also common in clinical practice $(4,11)$. For example, cavity expansion of syringomyelia can affect both the dorsomedial and ventromedial nerve, which further affects back muscles that control trunk balance and finally contributes to scoliosis (12). Spinal cord ischemia caused by tethering can induce abnormal sensory pathways and asymmetric paraspinal muscle strength, which finally leads to scoliosis. Other researchers have suggested that scoliosis is one of the clinical manifestations of intraspinal abnormalities $(4,7)$.

The typical treatment for these patients is two-stage surgery. However, this surgical method is associated with a number of limitations, including increased bleeding, increased duration, damage to normal tissue. In order to solve its problems, simultaneous surgical treatment in scoliosis associated with intraspinal abnormalities has gradually come into being. When compared to the classical surgery strategy, one-stage surgery is safer, more effective and with fewer apparent complications. The average surgical time, interoperative blood loss and hospital stay time associated with this procedure are reduced compared with traditional surgery. Better correction of scoliosis in single-stage surgery has also been reported in several studies. In the current study, the surgical time, blood loss and hospital stay time were similar to those in previous studies of one-stage surgery $(3,6)$. Following surgery, an improvement in both scoliosis and kyphosis was observed in all 12 patients, and no deterioration of neurological dysfunction was observed. The muscle strength of the lower extremities of the 10 patients and sphincter dysfunction has been improved. No significant loss of correction was observed during the follow-up time. Thus, one-stage surgery under neuroelectrophysiological monitoring was recommended for patients with intraspinal abnormalities and scoliosis.

Although spine deformity and intraspinal abnormalities are congenital diseases, scoliosis and neurological dysfunction appear relatively late (3). In the present study, the average age of the 12 patients were $29.5 \pm 13.7$ years, older than a mean age of 9.5 years reported by Mehta et al (6) and a mean age of 13 years reported by Hamzaoglu et al (2). Some of the patients did not visit the clinic once they had developed neurological dysfunction, but came to seek medical consultations after the deterioration of symptoms or an improvement in their financial situation, which revealed that both clinical symptoms and social factors can delay the 

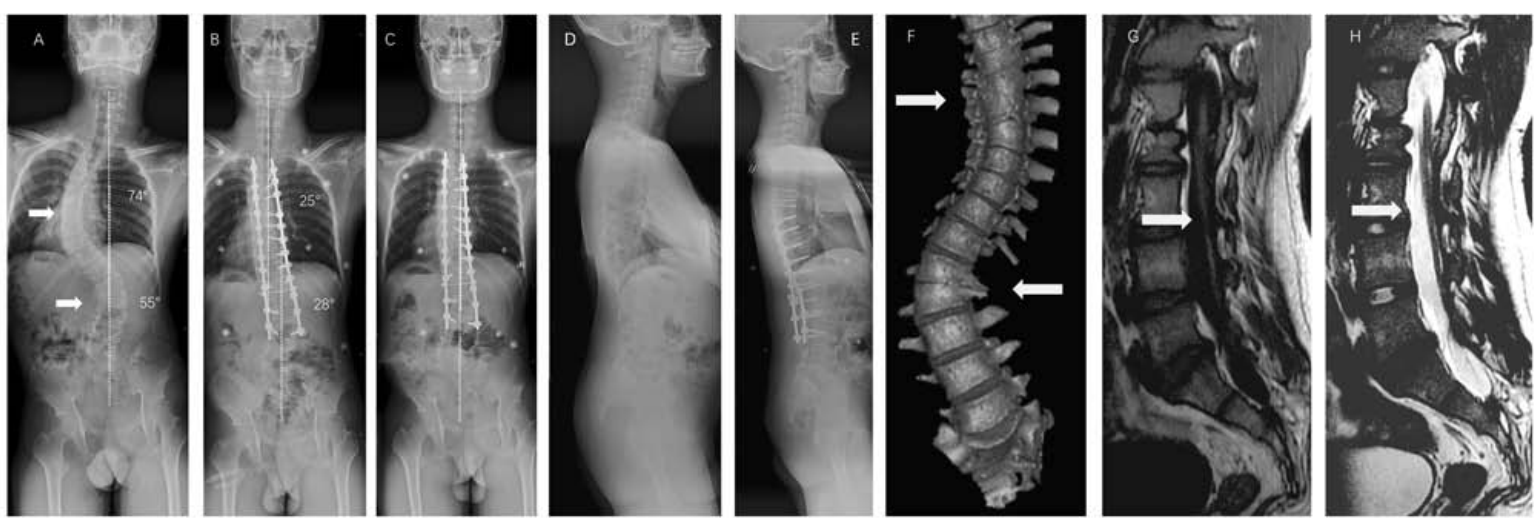

Figure 2. Radiographic images of the spine of a 17-year-old male patient with congenital scoliosis associated with tethered cord, taken pre- and postoperatively. (A) Preoperative image. The upper white arrow and lower white arrow indicate the thoracic curve and lumbar curve, respectively. (B) Postoperative image without shoe insert. (C) Postoperative image with shoe insert. Sagittal full-length image (D) before and (E) after surgery. (F) Preoperative 3D-CT of the spine. White arrows indicate failure of segmentation of T5-T7 and L1-L2, and wedged vertebrae of T6. (G) Preoperative sagittal T1WI MRI. White arrow indicates a tethered spinal cord with a low-lying conus at the level of L3-L4. (H) Preoperative sagittal T2WI MRI. The white arrow indicates a tethered spinal cord with a low-lying conus at the level of L3-L4.
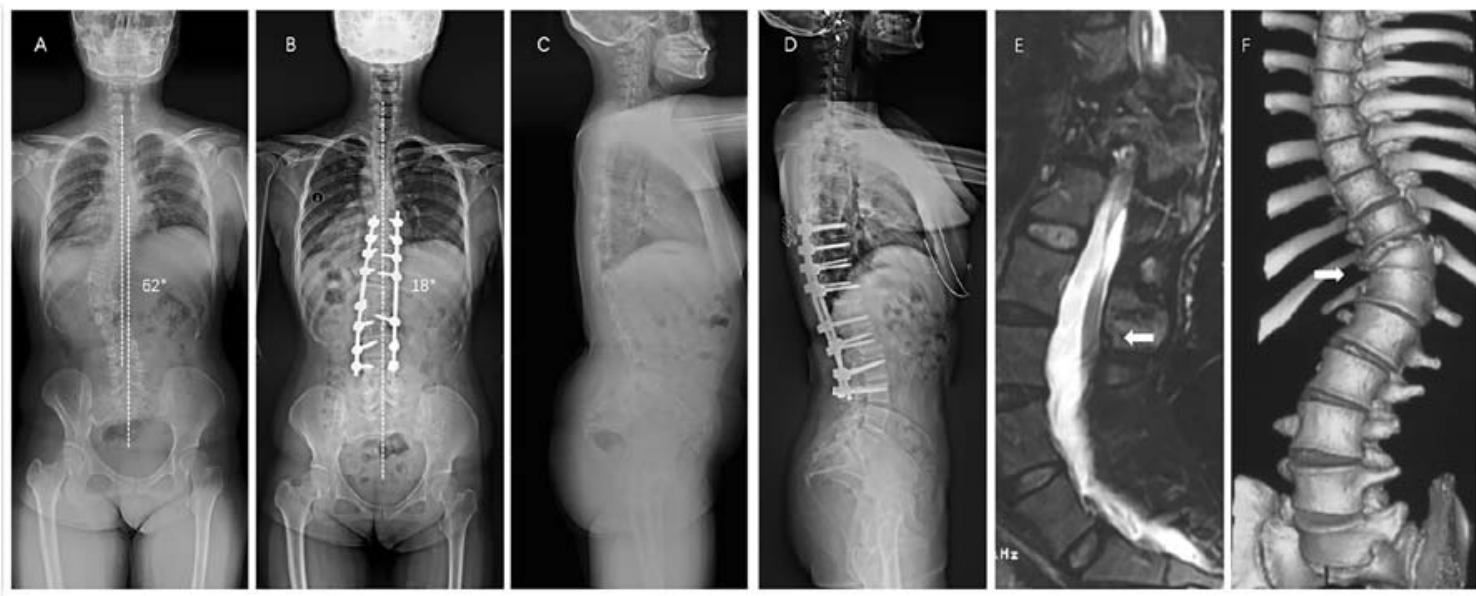

Figure 3. Radiographic images of the spine of a 27 -year-old female patient with congenital scoliosis associated with tethered cord, taken pre- and postoperatively. (A) Preoperative image in the coronal plane. (B) Postoperative image in the coronal plane with angle measurement. (C) Preoperative and (D) postoperative images in the sagittal plane. (E) Preoperative sagittal T2WI MRI. The white arrow indicates a tethered spinal cord with a low-lying conus at the level of L3. (F) Preoperative 3D-CT of the spine. White arrows indicate failure of segmentation of T11-T12, and wedged vertebrae of T11.
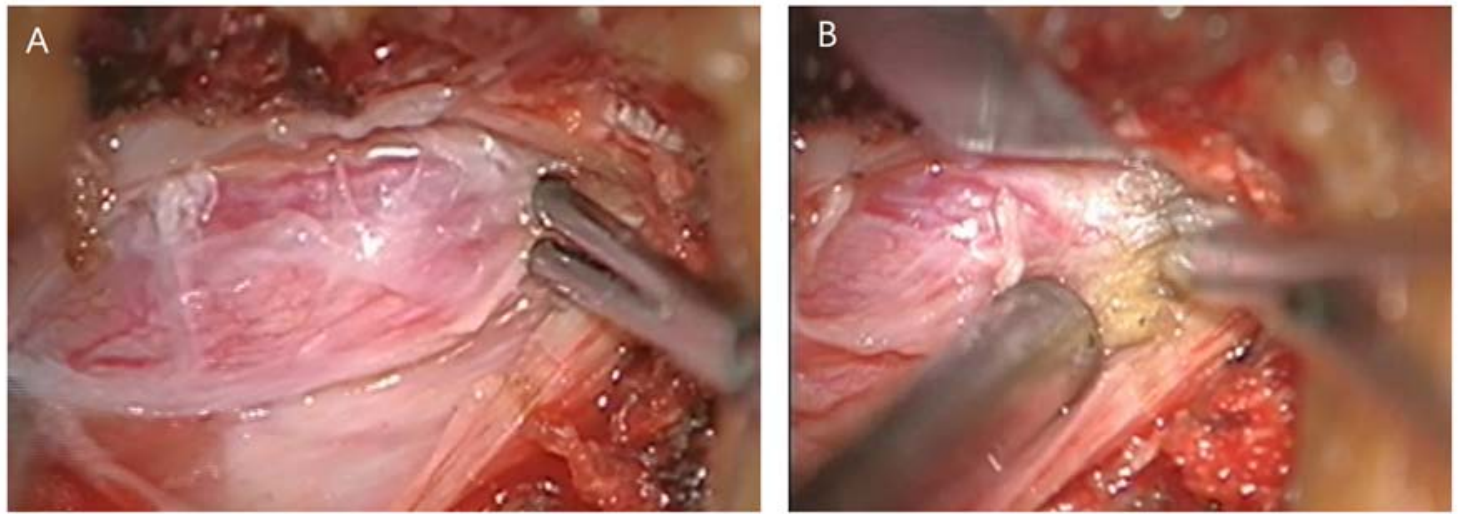

Figure 4. Images taken during surgery of case no. 12. (A) Electrophysiological monitoring confirmed the absence of nerves. (B) Cutting off the terminal filaments.

seeking of medical help (3). Due to this delay in seeking medical help and the mature skeletal development in the
12 patients in the present study, increased stiffness was observed in these patients compared with younger patients 
with scoliosis (Risser sign was level 2 in 1 patient, level 3 in 2 patients and level 5 in 9 patients). In addition, dysplasia was common in the congenital scoliosis patients (1 wedge-shaped vertebra, 1 malsegmentation, 1 wedge-shaped vertebra with malsegmentation and 2 hemivertebra with malsegmentation). Thus, it was difficult to perform corrective surgery in these patients. Osteotomy is a good choice for patients with stiff, severe scoliosis and kyphosis (13-15). In the current study, Schwab grade 2 osteotomy and spine release under a microscope were performed in all 12 patients to increase spinal flexibility and acquire better correction. For patients with hemivertebra and malsegmentation, further Schwab grade 3 or 4 osteotomy was performed to release the front column and resect the hemivertebra. Following surgery, scoliosis and kyphosis were improved by $67 \pm 11$ and $56 \pm 11 \%$, respectively. Coronal correction was better than the $27 \%$ reported by Mehta et al (6) and 23\% reported by Isu et al (16). In the present study, Schwab grade 2 osteotomy was performed under the operating microscope to release the spine, increase the flexibility of the spine and to achieve improved orthopedic effects, including correcting scoliosis and improving activity.

Scoliosis usually causes abnormalities in other parts of the body, including unequal shoulder height and pelvic asymmetry, which can not only change the local anatomy of the spine but also affect the balance and symmetry of the whole body. The pelvis, which is known as the mechanical support foundation of the human trunk, plays an important role in spinal balance and compensatory function (17). There is a correlation between pelvic rotation and lumbar curvature, and thoracic curvature; it can also be compensated for through lumbar spine-mediated coordination (18). Due to pelvic tilt and rotational decompensation, different degrees of trunk deviation will occur following thoracic curvature orthopedic internal fixation. Although pelvic reimbursement will eventually reach a balance during follow-up, this compensation mechanism is also constrained by spinal flexibility, and compensation for patients with rigid scoliosis is limited (19). Correcting the lumbar curve in the setting of pelvic obliquity may generate postoperative coronal decompensation. In patients with pelvic obliquity in which the spine is flexible and aligned, full correction of the curve may lead to significant coronal decompensation, as the scoliotic curve may be compensatory (20). If the spine is flexible, a shoe lift may yield a considerable amount of spinal coronal correction; however, if the spine is rigid, surgery is probably necessary, as is a shoe insert depending on the final standing alignment (21). Side bending and kyphosis were serious in the patients enrolled in the present study, which resulted in different degrees of rotation and tilt of the pelvis. Some patients had severe lumbar bending and pelvic tilt rotation. If lumbar bending is fused, it must be fixed to $\mathrm{S} 1$ or even the pelvis. The flexibility and compensatory ability of the waist will be completely lost, and the decompensation of the pelvis will aggravate the trunk tilt. Therefore, for this group of patients, we did not fully fuse lumbar bending during the operation. Following the operation, patients were asked to wear insoles to correct the pelvic tilt. Postoperative re-examination showed that the lumbar curves of the patients were partially compensated. The orthopedic effect was satisfactory and there was no serious deviation of the trunk.
Previous studies have reported that spinal canal lesions were mainly tethered cord, longitudinal cord, lipoma or meningocele $(22,23)$. However, in the present study, spinal canal lesions also included a variety of intraspinal canal tumors. However, this study had several limitations. First, this was a retrospective study. Second, the sample size was relatively small. Thus, further study of more patients and a longer follow-up time is needed to investigate the loss of correction and compensation of the pelvis.

In conclusion, single-stage surgery for scoliosis combined with intraspinal abnormalities was safe and effective, and can produce satisfactory correction of deformities and an improvement of neurological function. No postoperative complications were observed. The following factors are required for single-stage surgery for scoliosis combined with intraspinal abnormalities: i) The surgical team is experienced in dealing with scoliosis and intraspinal scoliosis at the same time; ii) a detailed plan is made before surgery; and, iii) interoperative neuroelectrophysiological monitoring is available.

\section{Acknowledgements}

Not applicable.

\section{Funding}

No funding was received.

\section{Availability of data and materials}

The datasets used and/or analysed during the current study are available from the corresponding author on reasonable request.

\section{Authors' contributions}

KW conceived, designed and analyzed the data. KW and FS performed all the simultaneous surgical treatments in scoliosis associated with intraspinal abnormalities. FS analyzed the data. HW conceived and designed the research and performed the surgeries. FS performed the research. FZJ analyzed the data and provided final approval of the version to be published. All authors read and approved the final manuscript.

\section{Ethics approval and consent to participate}

The current study was approved by the ethics committee of the Xuanwu Hospital of Capital Medical University (approval no. 2016020; V1.3, 2016-9-25; Beijing, China) and all the patients or their families gave informed consent and signed a statement to that effect.

\section{Patient consent for publication}

Not applicable.

\section{Competing interests}

The authors declare that they have no competing interests. 


\section{References}

1. Pereira EAC, Oxenham M and Lam KS: Intraspinal anomalies in early-onset idiopathic scoliosis. Bone Joint J 99-B: 829-833, 2017.

2. Hamzaoglu A, Ozturk C, Tezer M, Aydogan M, Sarier M and Talu U: Simultaneous surgical treatment in congenital scoliosis and/or kyphosis associated with intraspinal abnormalities. Spine (Phila Pa 1976) 32: 2880-2884, 2007.

3. Halawi MJ, Lark RK and Fitch RD: Neuromuscular scoliosis: Current concepts. Orthopedics 38: e452-e456, 2015.

4. Jankowski PP, Bastrom T, Ciacci JD, Yaszay B, Levy ML and Newton PO: Intraspinal pathology associated with pediatric scoliosis: A ten-year review analyzing the effect of neurosurgery on scoliosis curve progression. Spine (Phila Pa 1976) 41: 1600-1605, 2016.

5. Mcmaster MJ: Occult intraspinal anomalies and congenital scoliosis. J Bone Joint Surg Am 66: 588-601, 1984.

6. Mehta VA, Gottfried ON, McGirt MJ, Gokaslan ZL, Ahn ES and Jallo GI: Safety and efficacy of concurrent pediatric spinal cord untethering and deformity correction. J Spinal Disord Tech 24 401-405, 2011

7. Samdani AF, Asghar J, Pahys J, D'Andrea L and Betz RR: Concurrent spinal cord untethering and scoliosis correction: Case report. Spine (Phila Pa 1976) 32: E832-E836, 2007.

8. Little DG and Sussman MD: The risser sign: A critical analysis. J Pediatr Orthop 14: 569-575, 1994.

9. Ranu H, Wilde M and Madden B: Pulmonary function tests. Ulster Med J 80: 84-90, 2011.

10. Wang Z, Liu J, Shen J, Xue X and Qiu G: Abnormalities associated with congenital scoliosis: A retrospective study of 226 Chinese surgical cases. Spine (Phila Pa 1976) 38: 814-818, 2013.

11. Prahinski JR, Polly DW Jr, Mchale KA and Ellenbogen RG Occult intraspinal anomalies in congenital scoliosis. J Pediatr Orthop 20: 59-63, 2000.

12. Kontio K, Davidson D and Letts M: Management of scoliosis and syringomyelia in children. J Pediatr Orthop 22: 771-779, 2002.

13. Liu ZD, Li XF, Zang WP, Wang ZY and Wu LM: Combined pedicle subtraction osteotomy and polysegmental closing wedge osteotomy for correction of the severe thoracolumbar kyphotic deformity in ankylosing spondylitis. Zhonghua Wai Ke Za Zhi 47: 681-684, 2009 (In Chinese).

14. Gokcen B, Yilgor C and Alanay A: Osteotomies/spinal column resection in paediatric deformity. Eur J Orthop Surg Traumatol 24 (Suppl 1): S59-S68, 2014.
15. Holewijn RM, Schlösser TP, Bisschop A, van der Veen AJ, Stadhouder A, van Royen BJ, Castelein RM and de Kleuver M: How does spinal release and ponte osteotomy improve spinal flexibility? The law of diminishing returns. Spine Deform 3: 489-495, 2015.

16. Isu T, Chono Y, Iwasaki Y, Koyanagi I, Akino M, Abe H, Abumi K and Kaneda K: Scoliosis associated with syringomyelia presenting in children. Childs Nerv Syst 8: 97-100, 1992.

17. Skalli W, Zeller RD, Miladi L, Bourcereau G, Savidan M, Lavaste $\mathrm{F}$ and Dubousset J: Importance of pelvic compensation in posture and motion after posterior spinal fusion using CD instrumentation for idiopathic scoliosis. Spine (Phila Pa 1976) 31: E359-E366, 2006.

18. Gum JL, Asher MA, Burton DC, Lai SM and Lambart LM: Transverse plane pelvic rotation in adolescent idiopathic scoliosis: Primary or compensatory? Eur Spine J 16: 1579-1586, 2007.

19. Asher MA, Lai SM, Carlson BB, Gum JL and Burton DC: Transverse plane pelvic rotation increase (TPPRI) following rotationally corrective instrumentation of adolescent idiopathic scoliosis double curves. Scoliosis 5: 18, 2010.

20. Radcliff KE, Orozco F, Molby N, Chen E, Sidhu GS, Vaccaro AR and Ong A: Is pelvic obliquity related to degenerative scoliosis? Orthop Surg 5: 171-176, 2013.

21. Ames CP, Smith JS, Scheer JK, Bess S, Bederman SS, Deviren V, Lafage V, Schwab F and Shaffrey CI: Impact of spinopelvic alignment on decision making in deformity surgery in adults: A review. J Neurosurg Spine 16: 547-564, 2012.

22. Herman JM, Mclone DG, Storrs BB and Dauser RC: Analysis of 153 patients with myelomeningocele or spinal lipoma reoperated upon for a tethered cord presentation, management and outcome. Pediatr Neurosurg 19: 243-249, 1993.

23. Peng $\mathrm{L}$ and $\mathrm{Xu} \mathrm{BT}$ : Microsurgical treatment of complicated tethered cord resulting from mixed lipoma in a 12-year-old patient: A case report. Nan Fang Yi Ke Da Xue Xue Bao 31: 834-835, 2011 (In Chinese).

cc) (i) $\ominus$ This work is licensed under a Creative Commons EY NC ND Attribution-NonCommercial-NoDerivatives 4.0 International (CC BY-NC-ND 4.0) License. 